\title{
Predictive Factors for Obstructive Sleep Apnea: Epidemiological Study in A Romanian Population
}

\author{
Adelina Anton $^{1 *}$, Comsa Gheorghe Ionel ${ }^{1,4}$, Petronela-Ariadna Fildan ${ }^{1,3}$, Elena Dantes ${ }^{1,3}$, Oana \\ Cristina Arghir ${ }^{1,3}$ and Doina Ecaterina Tofolean ${ }^{1,2}$ \\ ${ }^{1}$ Faculty of Medicine, “Ovidius” University, Constanta, Romania \\ ${ }^{2}$ Department of Pneumology, County Emergency Clinical Hospital, Constanta, Romania \\ ${ }^{3}$ Department of Pneumology, Clinical Pneumophtisiology Hospital, Constanta, Romania \\ ${ }^{4}$ Department of Otorhinolaryngology, County Emergency Clinical Hospital, Constanta, Romania \\ *Corresponding author: Adelina Anton, Faculty of Medicine, “Ovidius” University, Constanta, Romania, \\ E-mail: adelinamiu@gmail.com
}

\section{ARTICLE INFO}

Received: 幽 September 03, 2020

Published: 幽 September 11, 2020

Citation: Adelina Anton, Doina Ecaterina Tofolean, Petronela-Ariadna Fildan, Elena Dantes, Oana Cristina Arghir, Comsa Gheorghe Ionel. Predictive Factors for Obstructive Sleep Apnea: Epidemiological Study in A Romanian Population. Biomed J Sci \& Tech Res 30(2)-2020. BJSTR. MS.ID.004935.

Keywords: Predictive Factors; Obstructive Sleep Apnea; Anthropometric Variables

\begin{abstract}
Introduction:Obstructive sleep apnea (OSA), a pathology increasingly prevalent, is a complex treatable syndrome that impact morbidity and mortality with serious cardiovascular and metabolic consequences. The interest for this pathology has increased in last decades although the syndrome remains underdiagnosed. Therefore, we aimed to identify the main predictive factors and to assess the interrelationship between them and OSA severity.
\end{abstract}

Methods: To this end, we performed a prospective study in a Romanian population. The study occurred for 48 months, between October 2015-september 2019, in the Sleep Disorders Center from County Emergency Clinical Hospital of Constanta. Polygraphy, blood tests, anthropometric parameters, demographic aspects were recorded for each subject. Predictive factors were disclosed using multivariate regression analysis.

Results:198 patients who referred to the Sleep Disorders Center and had been evaluated for OSA were enrolled. The study population included 147 male and 51 female; age range 27-82 years old. Among the 198 individuals, 43.5\% (86 patients) had moderate OSA and 56.5\% (112 patients) had severe OSA. Neck circumference, waist-hip ratio, BMI, gender, age was found to be significant predictors for OSA. In addition, $>50 \%$ of patients had metabolic alterations or had cardiovascular disorders.

Conclusion: No single factor was usefully predictive. Patients with OSA were more likely to be male, older, obese, have a thick neck, have a significant greater prevalence of habitual snoring and excessive daytime sleepiness, have hypertension or metabolic changes.

\section{Introduction}

Sleep medicine is a field in continuous development, sleep disorders breathing representing a structure that comprises a variety of respiratory disturbances that occur during sleep [1,2]. Obstructive sleep apnea syndrome (OSA) is the most frequent sleep breathing disorder with an increasing prevalence in last decades, but largely underdiagnosed and undertreated. It is characterized by recurrent episodes of obstruction of upper airways leading to sleep fragmentation and intermittent hypoxia during sleep. On one hand, OSA is a complex entity that has serious cardio-metabolic complications [1,2] with a severe impact on the patient's quality of life and requires multidisciplinary approach , on the other hand, it is a treatable pathology with an important influence on morbidity and mortality [3]. 
In Romania, this syndrome is still insufficiently well-known and studied with a variable prevalence in adult population due to differences in defining and diagnostic methods. However, the increasing number of sleep centers and the increasing number of patients who undergo a sleep study are raising awareness of this pathology but diagnostic test for suspected patients are limited due to cost and availability. Therefore, it is important to have a wellfounded approach to recognize patients with a high probability of being diagnosed with OSA. We hypothesis that identifying the main predictors for OSA will lead to the development of prediction formula that will help prioritizing patients to sleep study and treatment by the probability of severity of the disease.

\section{Aim}

The purpose of this study was to identify the main factors that are predictors for OSA and correlate them with disease severity in order to develop a model that disclose a role in diagnosing this sleep disorder.

\section{Material and Method}

We performed a prospective study in patients that were evaluated for OSA in the Sleep Disorders Center from County Emergency Clinical Hospital of Constanta, between October 2015 September 2019. Patient group consisted in subjects who referred to our Sleep Laboratory for evaluation of OSA or subjects that were referred by otolaryngologists with a high suspicion of sleep pathology. 257 consecutive individuals were investigated and each patient has signed the informed consent. All patients completed the study chart (which included general information like age, sex, occupation, use of alcohol, smoking and self-reported symptoms like snoring, stopping breathing, sleepiness), sleep questionnaire (Epworth Sleepiness Scale) and had a clinical evaluation with measurement of anthropometric variables like weight, height, BMI, neck circumference, waist circumference, waist-hip ratio. Furthermore, several biological markers were evaluated (glucose, cholesterol profile), blood pressure was measured and otorhinolaryngology examination (with fibroscopy of the nasal and pharyngeal cavity and Mallampati score assessment) was performed for everyone.

Patient's medical records were used to document comorbidities (cardiovascular, metabolic, respiratory) or chronic treatments. In addition, the presence of metabolic syndrome was evaluated using the National Heart, Lung, and Blood Institute (NHLBI) and the American Heart Association (AHA) guidelines. Metabolic syndrome was diagnosed if the patients met 3 out of the 5 criteria:

a. abdominal obesity (waist circumference $\geq 94 \mathrm{~cm}$ in men or $\geq 80 \mathrm{~cm}$ in women),

b. a low level of highdensity lipoprotein cholesterol $(<40 \mathrm{mg} /$ $\mathrm{dl}$ in men or $<50 \mathrm{mg} / \mathrm{dl}$ in women) or receiving lipid-lowering drug therapy, c. elevated triglyceride level $(\geq 150 \mathrm{mg} / \mathrm{dl})$ or receiving drug therapy for hypertriglyceridemia,

d. elevated blood pressure (systolic $\geq 130 \mathrm{mmHg}$ or diastolic $>85 \mathrm{mmHg}$ ) or receiving drug therapy for hypertension,

e. fasting plasma glucose level $(\geq 100 \mathrm{mg} / \mathrm{dl})$ or receiving drug therapy for hyperglycemia. Overnight respiratory polygraphy study was conducted in each case and every record was validated manually.

The following parameters were obtained: nasal airflow was registered trough a cannula, thoracic and abdominal movements were registered by using plethysmography belts, oxygen saturation and heart rate were measured by a finger probe oximeter, body position and number of snoring events were assessed by using sensors situated on the chest.

According to American Academy of Sleep Medicine definition, apnea was scored when the airflow signal drop by $80 \%$ or greater with continuing respiratory effort with a duration of 10 seconds or more. Hypopnea was scored when the peak signal excursions drop by $\geq 30 \%$ of pre-event baseline using nasal pressure more than 10 seconds associated with $\mathrm{a} \geq 4 \%$ oxygen desaturation from pre-event baseline. Apnea-hypopnea index (AHI) consisted in the number of apneas and hypopneas per hour of total sleep time. OSA was graded using apnea-hypopnea index as follows: mild (AHI 5 - 15 events/h), moderate (AHI 15 - 30 events/h) and severe (AHI $\geq 30$ events/h). For patients with AHI $<5$ events/h OSA was ruled out. In addition, in our study, patients with mild OSA (AHI between 5 and 15 events/h) were excluded since polysomnography was not available in these cases for a definite diagnosis.

Patients that were eligible met the following inclusion criteria:

a) Age $\geq 18$ years old

b) No previous diagnosis of OSA

c) $\mathrm{AHI} \geq 15$ events/hour

d) No secondary cause of hypertension

e) No family dyslipidemia

f) No type 1 diabetes

g) Informed consent was signed

Statistical data were processed using IBM SPSS version 2.0 and Microsoft Excel. Qualitative data were characterized by frequency and percentage whilst quantitative variables were described using the mean and standard deviation. $\mathrm{P}$ value was considered statistically significant at 0.05 or less.

\section{Result}

The study flow diagram is presented in Figure 1. From the total number of patients investigated in the Sleep Laboratory the study 
cohort counted 198 patients. The subjects who did not met the inclusion criteria wereexcluded. The 198 subjects enrolled included $141(71.2 \%)$ men and 57 (28.7\%) women, with a male-to-female ratio of 2.4:1. The mean age in the study group was 54.09 years. The youngest patient was 25 years old and the oldest patient was of 82 years old. A significantly increased prevalence of OSA was shown, in our study, in persons older than 40 years compared with individuals aged 20-40 years.The study group comprises 89(44.9\%) individual with moderate OSA and 109 (55.1\%) individuals with severe OSA. The mean AHI was $35.4 \pm 21.1$ events/h in women and $39.1 \pm 24.3$ events/h in men. Mean oxygen desaturation index was $40.3 \pm 19.7$ for men and $36.7 \pm 18.2$ for women. No significant correlation was found between gender and AHI severity.

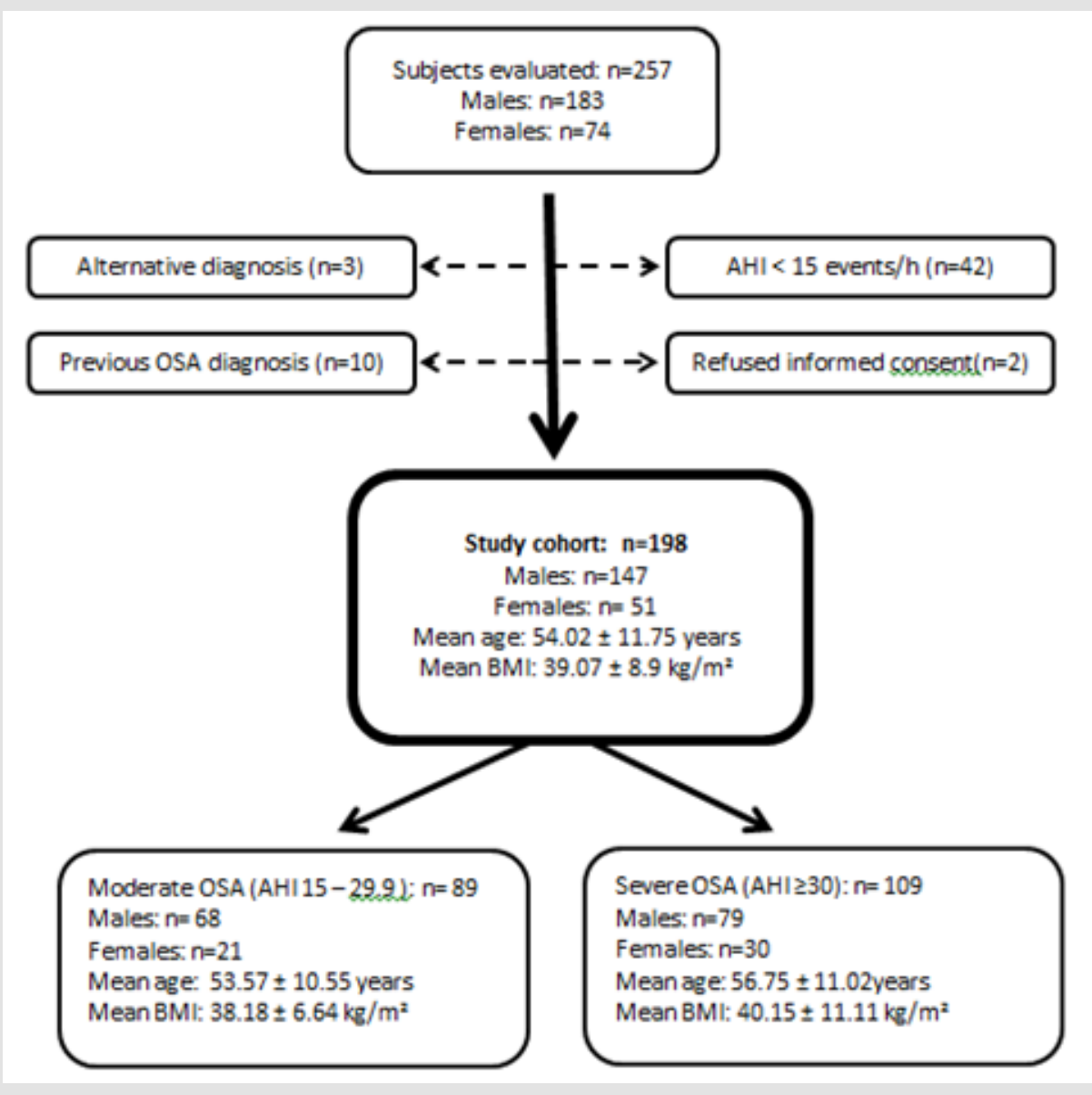

Figure 1: Demographic aspects of the study group (Abbreviations: OSA - obstructive sleep apnea, AHI - apnea-hypopnea index, BMI - body mass index).

In terms of demographic characteristic, another specific marker assessed was BMI. (Figure 2). The study has shown that most patients (84.8\%), regardless of gender, had a degree of obesity and only $13.1 \%$ were overweight. There was a small proportion $(2.02 \%)$ of patients who had normal BMI. However, BMI is not sufficient to evaluate the wide variation in body fat distribution, thus, additional measurements were made. Neck and waist circumference, waist-hip ratio was assessed for all subjects. In our cohort, the mean neck circumference was $45.6 \pm 4.9 \mathrm{~cm}$ in men and $39.5 \pm 4.2 \mathrm{~cm}$ in women. Regarding waist circumference the mean values were $109.2 \pm 14.5 \mathrm{~cm}$ for women and $118.6 \mathrm{~cm} \pm 13.2$ for men
Waist-hip ratio cut-off values used were $>0.9$ in men and $>0.85$ for women. WHR measurements showed a mean value of $1.01 \pm 0.24$ in male subjects and $0.9 \pm 0.11$ in female subjects. (Table 1) OSA has multiple ways of presenting, therefore, each patient enrolled in the study has reported several symptoms. We considered the following complaints that were related more frequently: snoring (68.6\%), stopping breathing (59.5\%), sleepiness (44.4\%), frequent awakening at night (23.2\%), nocturnal sweating (19.6\%) and morning headache (16.6\%).A significant association was revealed between snoring and $\mathrm{AHI}$ severity. 


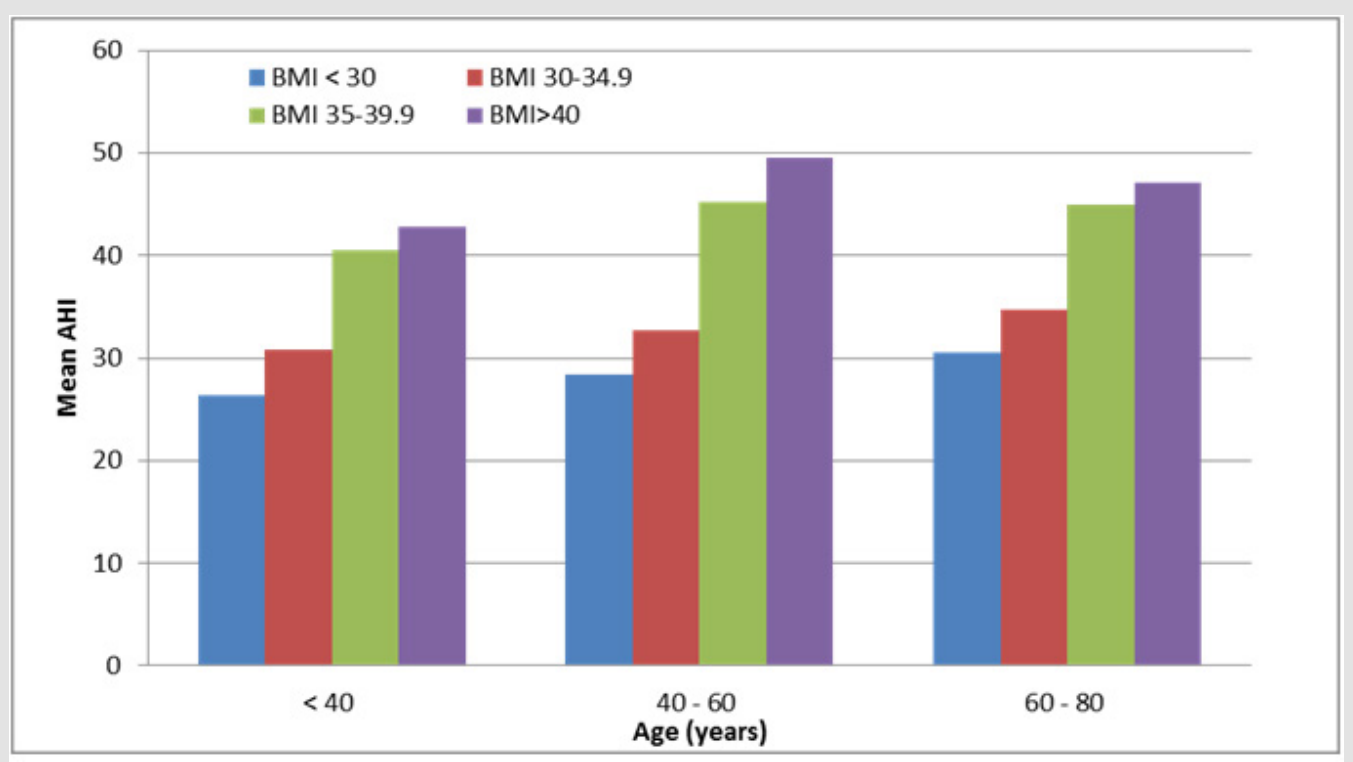

Figure 2: Correlation between mean AHI, BMI, age in the study group (Abbreviations: AHI - apnea hypopnea index, BMI body mass index).

Table 1: Characteristics of the group; Data are presented as mean $\pm S D$; Bold $p$ value is statistical significant (Abbreviations: OSA - obstructive sleep apnea, , BMI - body mass index, NC neck circumference, WC - waist circumference, WHR - waist-tohip ratio, NS refers to not significant $p$ value).

\begin{tabular}{|c|c|c|c|}
\hline & Men & Women & p-value \\
\hline Subjects (\%) & $141(71.2)$ & $57(28.7)$ & \\
\hline Age & $57.2 \pm 10.7$ & $53.5 \pm 8.7$ & NS \\
\hline BMI & $40.5 \pm 9.1$ & $36.4 \pm 7.5$ & NS \\
\hline NC & $45.6 \pm 4.9$ & $39.5 \pm 4.2$ & $<0.001$ \\
\hline WC & $118.6 \pm 13.2$ & $109.2 \pm 14.5$ & NS \\
\hline Waist-hip ratio & $1.01 \pm 0.24$ & $0.9 \pm 0.11$ & $<0.001$ \\
\hline
\end{tabular}

(Table 2) More than 50\% of enrolled patients had not reported excessive daytime sleepiness. Consequently, for an objective evaluation, Epworth Sleepiness Scale questionnaire was used for every patient. ESS was interpreted as follows: 0-5 points lower/ normal daytime sleepiness, 6-10 points higher normal daytime sleepiness, 11-12 points mild excessive daytime sleepiness, 13-15 points moderate excessive daytime sleepiness, 16-24 points severe excessive daytime sleepiness. The mean value of ESS score in our cohort was $9.4 \pm 4.3$ points. A significant correlation was found between high ESS score ( $>13$ points) and AHI severity. Several comorbidities are very frequently associated in patients with OSA. In our study population, most common associated diseases found were: Hypertension (78\%), Dyslipidemia (55\%), Metabolic syndrome (58\%), Type 2 diabetes (27\%), COPD/Asthma (19\%), ENT disorders (18\%) (Table 3).
Table 2: Related symptoms by gender category; Bold $p$ value is statistically significant.

\begin{tabular}{|c|c|c|c|}
\hline & Men (n=141) & Women (n=57) & p-value \\
\hline Symptoms & 99 & 37 & $<0.001$ \\
\hline Snoring & 93 & 25 & 0.08 \\
\hline Stopping breathing & 67 & 21 & 0.07 \\
\hline Sleepiness & 27 & 19 & 0.34 \\
\hline $\begin{array}{c}\text { Frequent awakening } \\
\text { at night }\end{array}$ & 15 & 24 & 0.74 \\
\hline Nocturnal sweating & 11 & 22 & 0.5 \\
\hline Morning headache & & & \\
\hline
\end{tabular}

Table 3: Comorbidities distribution in the study population; Bold $p$ value is statistically significant (Abbreviations: NS refers to not significant $p$ value)

\begin{tabular}{|c|c|c|}
\hline & Subjects (n) & p-value \\
\hline Comorbidities & 154 & $<0.001$ \\
\hline Hypertension & 108 & 0.31 \\
\hline Dyslipidemia & 53 & 0.24 \\
\hline Type 2 diabetes & 32 & NS \\
\hline CoPD & 5 & NS \\
\hline Asthma & 21 & NS \\
\hline Deviated nasal septum & 16 & NS \\
\hline $\begin{array}{c}\text { Chronic rhinitis } \\
\text { hyprbinate }\end{array}$ & 12 & NS \\
\hline
\end{tabular}




\section{Discussion}

Obstructive sleep apnea is a pathology that has a plurifactorial etiology. In our study, one objective was to identify the main predictors of OSA and correlate them with the severity of the syndrome. Firstly, the research highlighted that there is an increased incidence of OSA among male subjects. About two-thirds of enrolled patients were men, resulting a 2.4/1 male-to-female ratio. These observations are consistent with the literature, several studies had previously reported a male predominance in patients with OSA. Despite this finding, in the studied group, no correlation was found between sex and OSA severity, meaning that both women and men can have moderate or severe disease, regardless of gender. Another factor that had been considered is age. In our studied population, we have found that $76.76 \%$ of patients diagnosed with moderate or severe OSA were aged 40 years or older, with an increased prevalence as ageing. A peak had been emphasized for both women and men in the fifth and sixth decade of life. These findings are consistent with those reported in the literature, Malhotra et al. [4] explaining it through the age dependent changes that arise in the soft palate and pharynx.

Obesity is a major risk factor for OSA. In our study, most of patients were overweight or had different degrees of obesity. Evaluation of obesity included: BMI, NC, WC, WHR. The analysis of these metrics of obesity showed that the best unvaried predictor of AHI severity for each gender was waist-hip ratio, independently of BMI. In addition, for male subjects, a significant correlation between neck circumference and OSA severity was disclosed. In terms of symptoms, snoring was the most common manifestation reported for which patients were evaluated for a sleep disorder. Daytime sleepiness was assessed by using Epworth Sleepiness Scale questionnaire. Although the study comprises individuals with moderate and severe OSA, more than $50 \%$ of the patients did not report excessive daytime sleepiness and we concluded that, most frequently, sleepiness was underestimated. A significant correlation was found between snoring, BMI and moderate to severe ESS scores, but a singular predictor is only marginally useful.

Several studies had demonstrated the cardiovascular and metabolic implications of obstructive sleep apnea disclosing that the link between OSA and comorbidities can have serious consequences. In our cohort, most frequent newly diagnosed or pre-existent cardio-metabolic disorders were hypertension, metabolic syndrome, dyslipidemia and type 2 diabetes, out of which hypertension and MS have a strong correlation with AHI severity. Thus, the association needs to emphasize the role of OSA screening in patients with metabolic changes, hypertension and vice versa, considering that treating the sleep disorder has a positive impact on general outcome. In our study population, other comorbidities like COPD, Asthma and ENT pathologies were evaluated but no correlation with AHI severity was found. However, it is important to mention that ENT disorders can interfere with the treatment of OSA or can have a role in syndrome's physiopathology,therefore is mandatory to assess and treat these conditions.

\section{Conclusion}

OSA is a multifaceted disease with severe cardiovascular and metabolic repercussions which needs to be better understood and early diagnosed. Early identification of atrisk patients is still challenging, therefore recognizing these predictive factors and patterns should acknowledge the need of OSA screening prioritizing the access to sleep study (polygraphy / polysomnogram) for specific patients group.

The syndrome is a treatable condition therefore promptly recognition will not only improve the quality of life by its therapy, but also the life expectancy by meliorating the severity of the associated diseases.

\section{Acknowledgements}

The authors have no acknowledgements.

\section{Conflict of Interest}

No conflict of interest is to declare.

\section{References}

1. Ferran Barbé, Jean-Louis Pépin (2015) Obstructive Sleep Apnoea, Edited by Book, ISBN 978-1-84984-060-6.

2. Anita K Simonds, Wilfried de Backer (2012) ERS Handbook of Respiratory Sleep Medicine, European Respiratory Society, ISBN 978-184984-024-8.

3. Meir Kryger, Thomas Roth, William C Dement, (2017) Principles and Practice of Sleep Medicine, $6^{\text {th }}($ Edn), Elsevier, ISBN 978-0-323-24288-2.

4. Ohad Ronen, Atul Malhotra, Giora Pillar (2007) Influence of Gender and Age on Upper-Airway Length During Development, Pediatrics 120(4): e1028-e1034.

\section{ISSN: 2574-1241}

DOI: $10.26717 /$ BJSTR.2020.30.004935

Adelina Anton. Biomed J Sci \& Tech Res

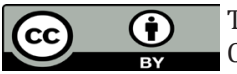

This work is licensed under Creative Commons Attribution 4.0 License

Submission Link: https://biomedres.us/submit-manuscript.php

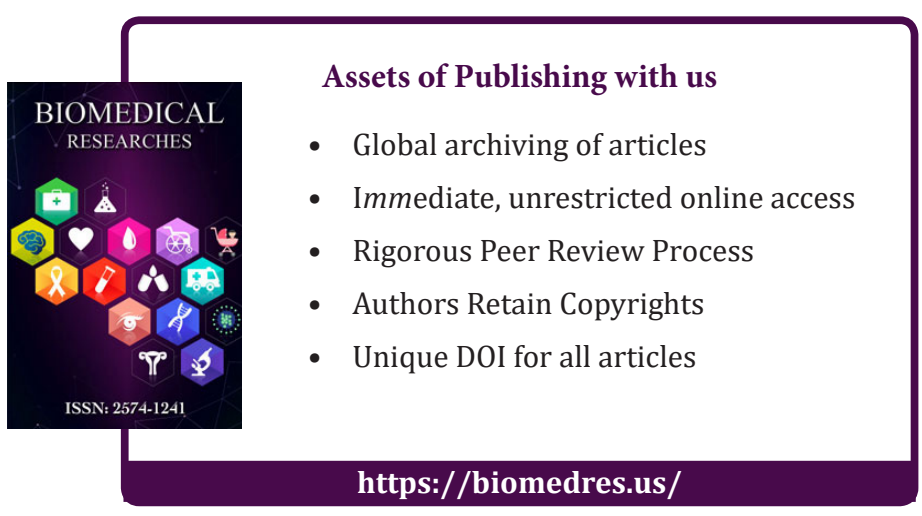

